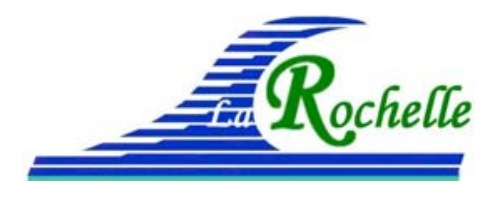
XVèmes Journées Nationales Génie Côtier - Génie Civil La Rochelle, 29 au 31 mai 2018

DOI:10.5150/jngcgc.2018.093 ～(C) Editions Paralia CFL

disponible en ligne - http://www.paralia.fr - available online

\title{
La base de données TEMPETES : un support pour une expertise collégiale et interdisciplinaire des informations historiques de tempêtes et de submersions
}

\section{Nathalie GILOY ${ }^{1}$, Claire-Marie DULUC ${ }^{1}$, Roberto FRAU ${ }^{2}$, Yann FERRET ${ }^{3}$, Thomas BULTEAU ${ }^{4}$, Franck MAZAS ${ }^{5}$, Thierry SAUZEAU ${ }^{6}$}

1. IRSN, BP 17, 92262 Fontenay-aux-Roses Cedex, France. nathalie.giloy-neodyme@irsn.fr

2. EDF LNHE, Laboratoire d'Hydraulique Saint-Venant, 6 Quai Watier, 78401 Chatou, France.roberto.frau@edf.fr

3. Shom, 13 rue du Chatellier, CS 92803, 29228 Brest Cedex 2, France. yann.ferret@shom.fr

4. BRGM, BRGM, F-33600 Pessac, France. t.bulteau@brgm.fr

5. ARTELIA, 6 rue de Lorraine, 38130 Echirolles, France. franck.mazas@arteliagroup.com

6. UPLC17, Forum des Marais Atlantiques, Quai aux Vivres, BP 40214, 17304 Rochefort Cedex, France.

thierry.sauzeau@univ-poitiers.fr

\section{Résumé :}

Au cours de la dernière décennie, la tempête Xynthia et d'autres ont marqué l'actualité de par leur intensité et les dégâts causés lors de leur passage sur le territoire français. Ces événements peuvent donner l'impression qu'ils n'ont été "jamais vu par le passé", mais le recensement des tempêtes plus anciennes $(1953,1987,1999 \ldots)$ montre que de tels phénomènes surviennent en fait régulièrement sur le littoral métropolitain. Dès lors, la collecte et l'analyse d'informations historiques apparaissent incontournables pour améliorer la prévention des risques littoraux, en particulier liée à la submersion marine. Afin de mutualiser les informations disponibles en France, une base de données relationnelle et spatiale a été développée au sein de l'IRSN. À ce stade, plus de 650 événements de tempête et/ou de submersion sur le littoral français et des pays voisins ont pu être recensés, sur une période s'étendant de 1500 à nos jours.

En parallèle à l'élaboration de cette base de données, un groupe de travail interdisciplinaire s'est constitué. Il intègre des ingénieurs, chercheurs, statisticiens et historiens appartenant à différents organismes. Un axe majeur de travail est l'intégration de nouvelles données historiques issues de diverses archives dans la base de données, ainsi que leur interprétation et la quantification des niveaux marins associés.

Mots-clés : Tempête, Submersion, Base de données, Surcotes, Niveaux marins. 


\section{Thème 7 - Risques côtiers}

\section{Introduction}

$\mathrm{Au}$ cours de la dernière décennie, les tempêtes Xynthia (février 2010), Carmen et Eleanor (janvier 2017), les tempêtes de l'hiver 2013-2014 et d'autres, ont marqué l'actualité de par leur intensité et les dégâts causés lors de leur passage sur le territoire français. Ces événements peuvent donner l'impression qu'ils n'ont été "jamais vu par le passé", mais le recensement des tempêtes plus anciennes (1953, 1987, 1999 par exemple) montre que de tels phénomènes, parfois qualifiés de "horsains" de par leur caractère exceptionnel, surviennent en fait régulièrement sur le littoral de la Manche et de l'Atlantique. Dès lors, la collecte et l'analyse d'informations historiques complémentaires apparaissent incontournables pour affiner l'estimation des aléas associés, en particulier la submersion marine, et ainsi améliorer la prévention des risques littoraux.

Des travaux de recensement d'événements de tempêtes historiques et susceptibles d'avoir provoqué des submersions existent déjà mais sont principalement menés à échelle régionale, dans le cadre par exemple d'élaboration de plans de prévention de risques naturels (PPRN) ou de projets de recherches divers (ROCHE et al., 2014, PERET \& SAUZEAU, 2014; GARNIER \& SURVILLE, 2012; ...). A échelle nationale, le projet de la base de données historiques sur les inondations (BDHI) mené dans le cadre de la directive européenne inondation a pour vocation de recenser et décrire "les phénomènes de submersions dommageables d'origine fluviale, marine, lacustre et autres survenues sur le littoral français" sous forme de fiche Document ou de fiche Inondation (LANG \& CEUR, 2014). Des projets dédiés aux submersions marines historiques et aux niveaux marins extrêmes à l'échelle nationale ont été menés par le BRGM et le Shom. Pour le BRGM il s'agissait, entre 2011 et 2013 (LAMBERT, 2011 ; GARCIN et al., 2011; LAMBERT \& GARCIN, 2013), de travailler sur la spécificité de recherche et d'analyse des submersions marines historiques antérieures aux années 1950 comme contribution à la BDHI. Les études menées au Shom sont principalement focalisées sur l'analyse des observations marégraphiques afin de quantifier ponctuellement les effets (surcotes) des événements de tempête majeurs du passé (DAUBORD et al., 2015), ou d'étudier l'évolution du niveau marin à l'échelle séculaire (FERRET, 2016). L'ensemble de ces études montre que le dépouillement de données d'archives ainsi que la numérisation et l'interprétation de données d'archives et marégraphiques est un travail fastidieux qui prend beaucoup de temps. Toutefois, les quelques valeurs extrêmes historiques reconstruites peuvent être intégrées dans des études statistiques des niveaux marins et sont précieuses pour améliorer les estimations des niveaux extrêmes (BULTEAU et al., 2015, HAMDI et al., 2015, FRAU et al., 2018). Outre-Manche, un projet de base de données de tempêtes et surcotes historique existe déjà sous le nom de "surgewatch" (HAIGH et al., 2017). 


\section{XVèmes Journées Nationales Génie Côtier - Génie Civil \\ La Rochelle, 29 au 31 mai 2018}

Lors des journées REFMAR, début février 2016, l'intérêt d'un groupe de travail pluridisciplinaire sur le sujet des submersions et tempêtes historiques a été souligné. Depuis, ingénieurs, chercheurs, statisticiens et historiens appartenant à différents organismes (IRSN, EDF, Shom, BRGM, ARTELIA, UPLC17, CEREMA) se réunissent au sein du groupe de travail "Tempêtes et Submersions historiques" (GT) dans le but de mutualiser les informations de tempêtes et submersions historiques au sein notamment d'une base de données commune.

D'autre part, pour répondre aux besoins d'expertise en sûreté nucléaire, l'IRSN a créé en 2015 une base de données reprenant les études et document disponibles et intégrant tous types de documents relatifs à des tempêtes ou des submersions historiques sur le littoral Manche et Atlantique. Cette base a été retenue par les membres du GT comme outil de mutualisation des apports de chacun, et s'intitule désormais BD TEMPETES. Une première version publique de la BD TEMPETES a été diffusée en 2017.

L'objectif de cet article est de présenter la BD TEMPETES et les travaux menés au sein du GT.

\section{Méthodologie}

La BD TEMPETES a été développée par l'IRSN avec le logiciel PostgreSQL. Ce logiciel open source de système de gestion de base de données relationnelle et objet (SGBDRO) permet la gestion de tables reliées entre elles et l'intégration de données spatiales via l'extension PostGIS ainsi que leur visualisation dans un logiciel de cartographie, QGis. La maintenance et la consultation de la base se fait via l'interface d'utilisation, pgAdminIII, en langage SQL (Structured Query Language).

Suite aux discussions au sein du GT, la base de données a légèrement évolué pour aboutir à la structure présentée sur la Figure 1. Elle contient six tables principales, chaque table possédant un identifiant clé, permettant par la suite de mettre ces tables en relation entre elles. La BD TEMPETES est organisée autour de la date (i.e. au moins les années d'occurrence) des événements de tempêtes et submersions, qui sont stockés dans la table événement, car chaque événement est susceptible de causer une submersion sur le littoral. La table source, directement reliée à la table événement, contient les informations sur chaque document intégré dans la BD TEMPETES, qui mentionne un événement, ainsi que le descriptif de cet événement. La table contributeur, rattachée à la table source, permet de tracer la personne ou l'organisme ayant mis la source à la disposition du consortium TEMPETES. Si l'information sur la localité du niveau marin recensé est disponible, cet événement sera référencé dans la table niveau marin. Les deux dernières tables sont deux tables spatiales, i.e. elles contiennent une référence spatiale dans un SIG. La table région contient les informations relatives aux régions et aux pays de la base de données, la table localité contient les informations géographiques des localités touchées par un événement. 


\section{Thème 7 - Risques côtiers}

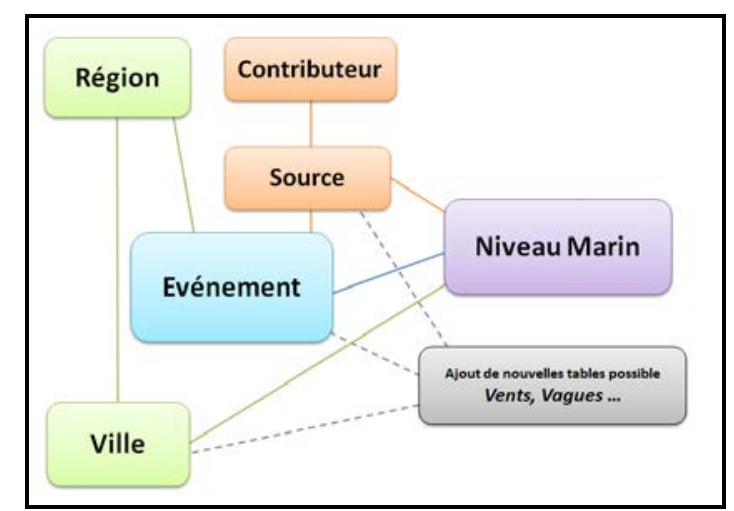

Figure 1. La structure de la BD TEMPÊTES.

Les données intégrées dans la BD TEMPETES sont entièrement publiques, la BD TEMPETES est soumise à la licence open source (https://opendatacommons.org/licenses/odbl/1.0/).

Dans le cadre de cette étude, tout ouvrage, rapport, document d'archive etc. mentionnant un événement est considéré comme une source.

La connaissance sur le déroulement des événements et de leurs impacts est fortement liée aux sources disponibles. Les sources textuelles intégrées dans la base de données sont la retranscription "brute" telle que recueillie dans le document source, c'est-à-dire qu'aucune interprétation de l'information de cette source n'est faite, qu'il s'agisse d'un document d'archive ou de données interprétées, voire reconstruites. Garder l'ensemble des informations "brutes" (descriptions originelles et interprétations/études ultérieures), permet à l'utilisateur de la BD TEMPETES de mener, si besoin est, une nouvelle enquête en tenant compte de l'ensemble des sources recensées concernant un événement.

Pour un événement donné, la qualité d'une source est variable, notamment s'il s'agit d'une source primaire ou secondaire: la source primaire est une observation, description ou mesure faite lors d'un événement par un témoin direct de l'événement, tandis qu'une source secondaire reprend des sources primaires ou les interprète. Lors de travaux menés dans le cadre d'une collaboration entre l'IRSN et Emmanuel Garnier (HAMDI et al., 2017 in review), une échelle de qualification des sources a été proposée et appliquée aux sources recensées dans la base :

1. La source est peu fiable (il est impossible de connaître l'origine exacte de l'information). Il s'agit notamment de certaines informations collectées sur Internet.

2. L'information est présentée dans des ouvrages techniques, sans clairement mentionner la source.

3. Des livres, rapports, journaux ou témoignages présentent des événements historiques en spécifiant clairement la source primaire.

4. L'information est présentée dans une source primaire, p.ex. un rapport original contemporain de l'événement. 


\section{XVèmes Journées Nationales Génie Côtier - Génie Civil \\ La Rochelle, 29 au 31 mai 2018}

Les sources de catégories les moins bonnes (qualité 1 ou 2) ne sont pas exclues du processus d'analyse des événements mais elles doivent être utilisées avec plus de précaution que les sources qualifiées en catégorie 3 voire 4, par exemple en cherchant systématiquement à les croiser avec ces dernières

Une distinction par type d'événement de tempête est également faite: lorsqu'une mention de submersion ou de brèche dans une digue existe, cet événement est considéré comme "submersion" ; lorsqu'aucune source ne fait mention d'une inondation ou d'une rupture de digue, l'événement est simplement dénommé "tempête", mais cet événement étant susceptible de causer une submersion.

\section{Résultats}

Au 15 février 2018, La BD TEMPETES contient 687 événements sur une période qui s'étend à ce jour du $16^{\mathrm{e}}$ au $21^{\mathrm{e}}$ siècle, cf. Figure 2, et contient 448 submersions et 237 tempêtes (sans mention de submersion) ayant touché le littoral Manche et Atlantique métropolitain.

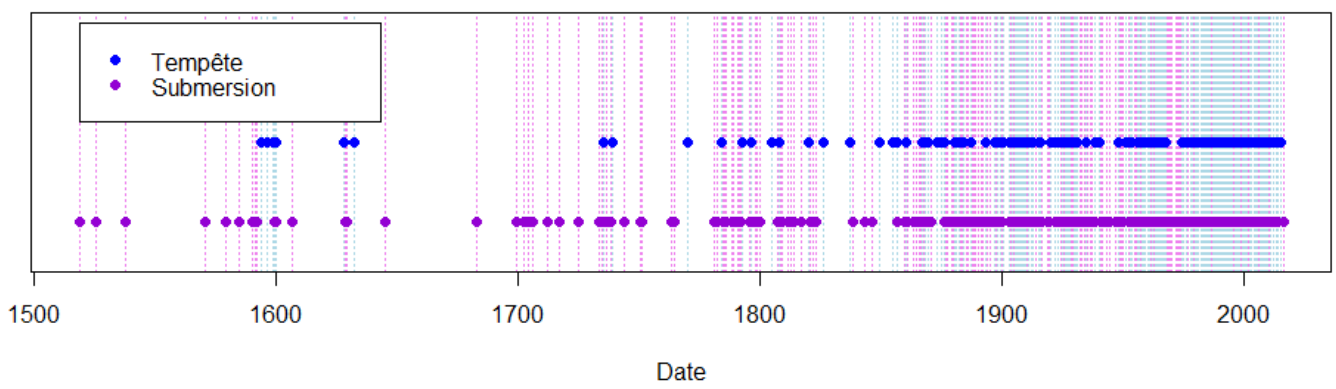

Figure 2. Frise temporelle des événements recensés dans la base de données TEMPETES selon leur type.

L'analyse de la frise temporelle indique une augmentation du nombre d'événements recensés, notamment due au fait que les événements les plus récents sont les plus documentées. Le nombre élevé d'événements de submersions par rapport à des tempêtes n'ayant pas provoqué de submersion peut s'expliquer par le fait que les recherches dans la littérature ou les archives sont très ciblées sur les submersions. Si on étendait cette moisson d'informations aux travaux menés sur les naufrages par (CABANTOUS, 1993) ou bien régionalement par (PERET, 2004), de nombreux nouveaux événements apparaitraient très vraisemblablement, sans que la corrélation avec le phénomène de submersion ne soit clairement établie.

La Figure 3 montre la qualité des sources par siècle, selon la classification des sources proposées dans (HAMDI et al., 2017 in review). Au total presque 1300 sources décrivent les 687 événements, et plus de la moitié des sources décrivent les événements 


\section{Thème 7 - Risques côtiers}

$\mathrm{du} 20^{\mathrm{e}}$ siècle. Il est également à noter que presque deux tiers des sources peuvent être considérés de bonne qualité (classées en catégorie 3, ou 4).

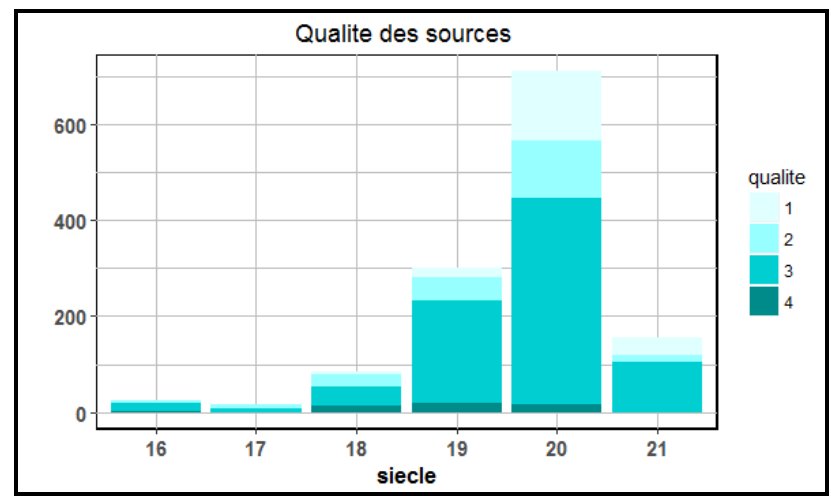

Figure 3. La qualité des sources (selon l'échelle proposée dans (HAMDI et al., 2017 in review) décrivant les événements recensés dans la base de données.

\section{Discussion}

La création de la BD TEMPETES met en évidence un nombre important d'événements de tempêtes et/ou de submersions depuis le $16^{\mathrm{e}}$ siècle (début de la période d'investigation). L'IRSN s'intéressant particulièrement aux données de niveau marin et de surcotes, une sélection d'information disponible est recopiée dans une table spécifique, permettant de capitaliser des valeurs numériques déjà estimées telles que la surcote de pleine mer et le niveau marin maximum observé dans des champs dédiés, permettant une extraction rapide, utile dans le cadre d'estimations statistiques.

La BD TEMPETES ne constitue évidemment pas un recensement exhaustif des événements survenus depuis le $16^{\mathrm{e}}$ siècle sur le littoral Manche et Atlantique. Par ailleurs, il est tout à fait possible d'élargir la zone d'étude à d'autres zones littorales ainsi que d'intégrer des événements qui ont eu lieu avant 1500.

La structure de la BD TEMPETES est assez simple et rend possible l'intégration d'éléments textuels. Des réflexions sont en cours pour permettre d'associer aux sources des images (photographies, cartes postales etc.). Par ailleurs, il est tout à fait possible d'ajouter des champs aux différentes tables ainsi que des nouvelles tables dédiées à d'autres variables détaillant par exemple le vent ou les vagues, ou les dégâts causées par un événement, en fonction des besoins et objectifs des utilisateurs.

Le dépouillement d'archives et la quantification de niveaux marins et de surcotes est un travail long et fastidieux donnant des résultats uniquement localisés. Avant l'apparition d'instruments de mesures vers la fin du $18^{\mathrm{e}}$ siècle, seules les narrations, qu'elles soient issues de journaux intimes, de registres paroissiaux, délibérations municipales etc., livrent des informations qualitatives sur les événements. Une analyse critique de chaque document et du vocabulaire utilisé, ainsi que le croisement des sources sont souvent nécessaires afin de pouvoir reconstruire le déroulement de l'événement dans son 


\section{XVèmes Journées Nationales Génie Côtier - Génie Civil \\ La Rochelle, 29 au 31 mai 2018}

contexte historique. De plus, pour pouvoir estimer des niveaux atteints pendant un événement, des informations complémentaires sont nécessaires comme par exemple des plans de localisation, ou d'infrastructures inondées.

Les partenaires du GT souhaitent disposer d'un outil disponible à court terme, permettant de disposer d'informations de niveaux marins et de surcotes historiques déjà identifiées. Ayant élaboré la BD TEMPETES, l'IRSN continuera dans un premier temps à assurer la gestion et la maintenance de la base ainsi que l'organisation du GT.

Enfin, un des objectifs du GT est de mettre en place une méthodologie d'analyse de données historiques permettant la quantification de niveaux marins historiques et de surcotes de pleine mer. Ces interprétations pourront être tracées sous forme de fiches événements qui seront également intégrées dans la base de données comme sources complémentaires.

\section{Conclusion et perspectives}

La BD TEMPETES a pour but de capitaliser tout type d'information sur les événements de tempêtes et de submersions historiques. Début 2018, plus de 650 dates d'événements ont été inventoriées, mais ce recensement est loin d'être exhaustif et est susceptible d'évoluer. Une échelle de qualification des sources a permis de montrer que, bien que celles-ci soient très rarement des sources primaires, leur qualité est souvent bonne. Quelle que soit leur qualité, les sources sont intégrées dans la BD TEMPETES telles que disponibles dans le type de document, laissant la possibilité à l'utilisateur d'en faire sa propre critique et sa propre analyse.

Un objectif à moyen-terme est de faciliter le renseignement ainsi que l'exploitation de la base de données, et de pouvoir intégrer des sources visuelles telles que des photographies d'archives, des anciennes cartes postales, etc. Cette base, dont la structure n'est pas figée, est une base open-source et disponible sur demande à l'IRSN.

En parallèle à l'élaboration de cette base de données un GT intégrant ingénieurs, chercheurs, statisticiens et historiens issues de différents organismes et travaillant sur les thématiques d'inondations marines historiques s'est constitué. Les membres partenaires disposent de la BD TEMPETES permettant des requêtes d'extraction des informations relatives à des événements historiques de tempêtes ou de submersions. Cette base pourra être complétée avec leurs informations propres et améliorée en fonction des retours des utilisateurs. Dans le même temps, un travail commun a démarré pour développer une démarche d'interprétation de données historiques, de quantification et de validation de niveaux marins / surcotes, ainsi que l'estimation des incertitudes associées. Ce travail pourra par exemple s'appuyer sur des études préliminaires de reconstruction de surcotes historiques menées par certains partenaires (HAMDI et al., 2017, in review; GILOY et al., 2017 ; REGNIER et al., 2017), mais dont certaines hypothèses nécessiteraient d'être confortées au sein du GT. En effet, l'élaboration d'une 


\section{Thème 7 - Risques côtiers}

démarche d'interprétation des données historiques et de quantification requiert l'expertise, multidisciplinaire, de l'ensemble des partenaires impliqués.

\section{Bibliographie}

BULTEAU T., IDIER D., LAMBERT J., GARCIN M (2015). How historical information can improve estimation and prediction of extreme coastal water levels: application to the Xynthia event at La Rochelle (France). Nat. Hazards Earth Syst. Sci., Vol. 15, pp 1135-1147. https://doi.org/10.5194/nhess-15-1135-2015

CABANTOUS A. (1993). Les côtes barbares. Pilleurs d'épaves et sociétés littorales en France (1680 - 1830). Fayard, Paris 311 p.

DAUBORD C., ANDRÉ G., GOIRAND V., KERNEIS M. (2015). Rapport technique final du projet NIVEXT. Rapport d'étude Shom, $448 \mathrm{p}$.

FERRET Y. (2016). Reconstruction de la série marégraphique de Saint-Nazaire. Rapport d'étude Shom, $122 \mathrm{p}$.

FRAU R., ANDREEWSKY M., BERNARDARA P. (2018). The use of historical information for regional frequency analysis of extreme skew surge. Accepté et en cours de publication sur Nat. Hazards Earth Syst. Sci.

GARCIN M., LAMBERT J., VINCHON C. (2011). Contribution au recensement des submersions marines historiques liées aux tempêtes sur le littoral français métropolitain - exploitation des fiches. Rapport BRGM/RP-60531-FR.

GARNIER E, SURVILLE F. (2010). La tempête Xynthia face à l'histoire. Le Croix Vif, Saintes. 176 p.

GILOY N., GARNIER E., HAMDI Y., DULUC C.-M. (2017). Reconstructing historic surges in the Dunkirk Area, France. Third international conference EVAN, 5-7 September 2017, Southampton (UK).

HAIGH I.D., OZSOY O., WADEY M.P., NICHOLLS R.J., GALLOP S.L., WAHL T., BROWN J.M. (2017). An improved database of coastal flooding in the United Kingdom from 1915 to 2016, Scientific Data 4, 1-10. http://dx.doi.org/10.1038/sdata.2017.100

HAMDI, Y., BARDET L., DULUC C-M., REBOUR V. (2015). Use of historical information in extreme-surge frequency estimation: the case of marine flooding on the La Rochelle site in France. Nat. Hazards Earth Syst. Sci, Vol. 15, pp 1515-1531. https://doi.org/10.5194/nhess-15-1515-2015

HAMDI Y., GARNIER E., GILOY N., DULUC C-M., REBOUR V. (2017, in review). Analysis of the risk associated to coastal flooding hazards: A new historical extreme storm surges dataset for Dunkirk, France. Nat. Hazards Earth Syst. Sci. Discuss. https://doi.org/10.5194/nhess-2017-417

LAMBERT J. (2011). Contribution au recensement des submersions marines historiques liées aux tempêtes sur le littoral français métropolitain. Rapport final. Rapport BRGM/RP-59937-FR. 


\section{XVèmes Journées Nationales Génie Côtier - Génie Civil \\ La Rochelle, 29 au 31 mai 2018}

LAMBERT J., GARCIN M. (2013). Submersions marines historiques. BRGM/RP62644-FR.

LANG M., CEUR D. (2014). Les inondations remarquables en France. Editions Quae, Versailles, $640 \mathrm{p}$.

PERET J. (2004). Naufrages et pilleurs d'épaves sur les côtes charentaises aux XVII et XVIII ${ }^{e}$ siècles. Geste Editions, La Crèche, 263 p.

PERET J., SAUZEAU T. (2014). Xynthia, ou la mémoire réveillée. Geste Editions, la Crèche, $296 \mathrm{p}$.

REGNIER F., FRAU R., ANDREEWSKY M., GILOY N. (2017). Increasing skew surge database with the collection of archaeological data, Third international conference EVAN, 5-7 September 2017, Southampton (UK).

ROCHE A., BARAER, F., LE CAM H., MADEC, T. GAUTIER, S., JAN, G., GOUTX, D. (2014). Projets VIMERS: une typologie des tempêtes bretonnes pour prévoir l'impact des tempêtes à venir et mieux s'y préparer. Journées Nationales Génie Côtier Génie Civil, Dunkerque, pp 925-932. http://dx.doi.org/10.5150/jngcgc.2014.102 
Thème 7 - Risques côtiers 